\title{
A Study of Beam Parameters Using NLSE in Chalcogenide Glass Through Variational Method with a Gaussian Trial Function
}

\author{
Chironjit Hazarika ${ }^{1, ~ *, ~ A b h i j e e t ~ D a s ², ~ S u b r a t a ~ H a z a r i k a ~}{ }^{2}$ \\ ${ }^{1}$ Department of Physics, Mariani College, Jorhat, Assam, India \\ ${ }^{2}$ Department of Physics, Assam University, Diphu Campus, Karbi Anglong, Assam, India
}

Email address:

chironjithazarika@gmail.com (C. Hazarika)

\section{To cite this article:}

Chironjit Hazarika, Abhijeet Das, Subrata Hazarika. A Study of Beam Parameters Using NLSE in Chalcogenide Glass Through Variational Method with a Gaussian Trial Function. American Journal of Optics and Photonics. Vol. 3, No. 4, 2015, pp. 43-47.

doi: 10.11648/j.ajop.20150304.11

\begin{abstract}
Using variational method for an elliptical Gaussian optical beam trial function, self -action in bulk chalcogenide glass (Kerr media) is investigated. Emphasis is laid on the study of variation in beam width, curvature, phase and intensity of the beam with propagation distance. Solutions predict stationary self-focusing of the elliptical beam and an effective beam collapse at $10 \mathrm{P}_{\mathrm{cr}}$ input power. These study is significant in the choice of parameters in optical communications.
\end{abstract}

Keywords: Self- Focusing, Kerr Nonlinearity, Gaussian Laser Beam

\section{Introduction}

Self- focusing, an optical self- action effect, occurs when the propagating electromagnetic field induces a change in the refractive index of the non-linear medium through which the field propagates. Self-focusing and self-trapping effects of laser pulses and beam can lower the threshold of other nonlinear processes like self-phase modulation, optical damage, regulate design of high energy lasers sources required for long - distance optical communication system [1], controlled laser fusion [2,3], coherent beam combining[4]and molecular dynamic diagnostics $[8,9]$ etc. These consequences of selfaction effects on the propagation of intense laser pulses and beams have attracted attention of researchers for more than fifty years now. Intense researches on laser propagation in nonlinear media and associated self-action effects lead to formulation of different theoretical concepts and mathematical models of self-focusing, beam self-trapping, filamentation, supercontinuum generation and filament plasma defocusing $[5,6,7,10]$. Starting with Chiao et. al.'s paper on self-trapped model of an intense laser beam in dielectric waveguide [11], which was an explanation to Hercher's experimental observation of first ever 'optical damage' in 1964 caused by a Q-switch laser in glass [12], more than 1500 research papers involving self-focusing and self-trapping in one way or other [1] have been published since the concept of laser beam self-focusing and selftrapping was proposed by Askaryan in 1962 [13]. Theoretically, the temporal and spatial electromagnetic-field behaviour of a propagating beam in nonlinear bulk media were investigated by several methods that includes beam propagation method (BPM) [14,15], nonlinear Schrödinger equation (NLSE) [16,17], finite-difference time-domain (FDTD) method [18,19] etc. Currently, frontier theoretical research on laser beam propagation in nonlinear medium deals with formulations and creative solutions of nonlinear Schrodinger equation (NLSE) that describes laser beam collapse, self-trapping, dispersion, filamentation, modulation instability, pulse splitting and other extraordinary particularities of nonlinear beam propagation [ 20, 21, 22]. In this communication propagation of intense elliptical Gaussian beam in chalcogenide glass with refractive index $\left(n_{0}=2.8\right)$ is investigated as the beam approches the self focusing distances $\left(\mathrm{z}_{\mathrm{sf}}\right)$ by variational method. Using Gaussian trial function, equations are derived for beam width, curvature, phase shift as a function of propagation distance to study their variations and interpretions of self action effects.

The present study characterizes its significance as currently non-linear effects are exploited at low optical powers in chalcogenide glasses. Due to their large intensity dependent refractive index and their low linear absorptions at optical communication wavelength makes these glasses 
attractive materials for use in a variety of optical devices $[23,24,27]$.Hence Study of the behaviour of the intense beam in chalcogenide glass at self-focusing distance can facilitated the choice of power, wavelength etc. and fabricate symmetric waveguides for efficient optical transmission.

This paper is organized as follows: in section 2, the theoretical formalism of the problem is outlined, section 3 is devoted to results and discussion and paper is concluded in section 4 .

\section{Basic Formulation}

The propagation of a light beam in a Kerr medium under the paraxial approximation can be described by the nonlinear Schrodinger equation,

$$
\left(\frac{\partial^{2}}{\partial x^{2}}+\frac{\partial^{2}}{\partial y^{2}}-2 i k \frac{\partial}{\partial z}\right) E+2 k^{2} \frac{n_{2}}{n_{0}}|E|^{2} E=0
$$

Where $\mathrm{k}$ is the linear wave vector, $\mathrm{x}$ and $\mathrm{y}$ are the transverse co-ordinates, $\mathrm{z}$ is the longitudinal coordinates, $n_{2}$ is the third order nonlinear coefficient and $n_{0}$ is the linear refractive index of the medium. This equation describes the slowly varying field inside the nonlinear medium. The first term and second term of equation (1) represents the diffraction of the beam in transverse direction and the last term works as a source to generate different modes of the electric field due to the nonlinear response of the medium. Kerr nonlinearity is one of the important nonlinear response occurring in glasses, gases etc. When the diffraction term comes into balance with the focusing due to Kerr nonlinearity then self-focusing of optical beam occur.

The solution of the variational problem

$$
\delta \iiint L d x d y d z=0
$$

also solves the nonlinear Schrodinger equation (1) with an appropriate Lagrangian L. Equation (1), is reformulated as a variational problem using the Lagrangian

$$
\mathrm{L}=\left|\frac{\partial E}{\partial x}\right|^{2}+\left|\frac{\partial E}{\partial y}\right|^{2}-i k\left(E \frac{\partial E^{*}}{\partial z}+E^{*} \frac{\partial E}{\partial z}\right)-\frac{n_{2} k^{2}}{n_{0}}|E|^{4}
$$

where ' $*$ ' denotes complex conjugation.

As a trial solution, the Gaussian field distribution function is considered for the procedure of the variational approach

$$
\begin{gathered}
\mathrm{E}(\mathrm{x}, \mathrm{y}, \mathrm{z})=A_{1}(\mathrm{z}) \exp \left[-\frac{x^{2}}{2 \alpha^{2}}-\frac{y^{2}}{2 \beta^{2}}\right] \exp \left[i \left\{x^{2} c_{1}(z)+\right.\right. \\
\left.\left.y^{2} c_{2}(z)+\phi(z)\right\}\right]
\end{gathered}
$$

where $A_{1}(\mathrm{z})$ and $\phi(z)$ are the amplitude and phase of the complex field $E(\mathrm{x}, \mathrm{y}, \mathrm{z}), \alpha$ and $\beta$ are the width parameters of the Gaussian beam in $\mathrm{x}$ and $\mathrm{y}$ direction respectively, $c_{1}(\mathrm{z})$ and $c_{2}(\mathrm{z})$ are the curvature parameters in $\mathrm{x}$ and $\mathrm{y}$ direction.

Inserting the trial function into equation (3), and integration with respect to $\mathrm{x}$ and $\mathrm{y}$ yield the effective Lagrangian $\langle L\rangle$.

$$
\langle L\rangle=\int_{-\infty}^{\infty} L d x d y=0
$$

$$
\langle L\rangle=\left\langle L_{O}\right\rangle+\left\langle L_{1}\right\rangle
$$

Where,

$$
\begin{gathered}
\left\langle L_{O}\right\rangle=2 \pi \alpha^{3} \beta A_{1}^{2}(z) c_{1}^{2}(z)+2 \pi \beta^{3} A_{1}^{2}(z) c_{2}^{2}(z) \\
+\frac{1}{2 \alpha} \pi \beta A_{1}^{2}(z)+\frac{1}{2 \beta} \pi \alpha A_{1}^{2}(z)-2 \pi \alpha \beta k A_{1}^{2}(z)\left[\frac{\alpha^{2}}{2} \frac{\partial c_{1}}{\partial z}\right. \\
\left.+\frac{\beta^{2}}{2} \frac{\partial c_{2}}{\partial z}+\frac{\partial \phi}{\partial z}\right] \\
\left\langle L_{1}\right\rangle=-\frac{\pi^{2} n_{2} \mathrm{~K}^{2}}{n_{0}} \alpha^{2} \beta^{2} A_{1}^{4}(z) .
\end{gathered}
$$

Now from the reduced variational principle, $\delta \int\langle L\rangle \mathrm{dz}=0$, i.e considering the beam propagation along $z$ direction, it is possible to derive variational equations with respect to $\alpha, \beta, A_{1}(z), c_{1}(\mathrm{z}), c_{2}(\mathrm{z}), \phi(z)$ etc. Using the procedure of $[25,26]$ and after some rearrangement, the following differential equations for $\alpha, \beta, c_{1}(\mathrm{z}), c_{2}(\mathrm{z})$ and $\phi(z)$ are obtained, which describe the evolution of the Gaussian beam in Kerr medium.

$$
\begin{aligned}
c_{1}(\mathrm{z}) & =-\frac{\mathrm{k}}{2 \alpha} \frac{d \alpha}{d z} \\
c_{2}(\mathrm{z}) & =-\frac{\mathrm{k}}{2 \beta} \frac{d \beta}{d z} \\
\frac{\partial \phi}{\partial z} & =\frac{1}{4 \mathrm{k}}\left[\frac{1}{\alpha^{2}}+\frac{1}{\beta^{2}}\right] \\
\frac{\partial c_{1}}{\partial z} & =\frac{k}{2 \alpha^{2}}\left(\frac{d \alpha}{d z}\right)^{2}-\frac{\mathrm{k} H_{0}}{\alpha^{2}} \\
\frac{\partial c_{2}}{\partial z} & =\frac{\mathrm{k}}{2 \beta^{2}}\left(\frac{\partial \beta}{\partial z}\right)^{2}-\frac{\mathrm{k} H_{0}}{\beta^{2}}
\end{aligned}
$$

where,

$$
H_{0}=\frac{\pi n_{2} \alpha \beta A_{1}^{2}(z)}{n_{0}}
$$

$c_{1}$ and $c_{2}$ only depends on $z$, So we can write $\frac{\partial C_{1}}{\partial z} \approx \frac{d C_{1}}{d z}$ and $\frac{\partial C_{2}}{\partial z} \approx \frac{d C_{2}}{d z}$.

Differentiating equation (6) and (7) and putting the values of $\frac{d C_{1}}{d z}$ and $\frac{d C_{2}}{d z}$ in equation (9) and (10) yield,

$$
\begin{aligned}
& \frac{d^{2} \alpha}{d z^{2}}-\frac{2 H_{0}}{\alpha}=0 \\
& \frac{d^{2} \beta}{d z^{2}}-\frac{2 H_{0}}{\beta}=0
\end{aligned}
$$

Equations (12) and (13) will be used for numerical analysis.

\section{Result and Discussion}

The finite difference scheme based on fixed point iteration method is used to obtain numerical solutions of equations (12) and (13) that describe the spatial evolution of beam width. Here the critical power is calculated using the relation [1].

The effective Lagrangian can be written as, 


$$
P_{c r=\frac{\pi \lambda^{2}(.61)^{2}}{8 n_{0} n_{2}}}
$$

The solutions are plotted in Fig. 1 and are for a beam propagating in chalcogenide glass of refractive index $\left(\mathrm{n}_{0}\right)=2.8$ and non-linear refractive index $\left(n_{2}\right)$ of $13 \mathrm{x}$ $10^{-14} \mathrm{~cm}^{2} / \mathrm{W}$ at three input powers viz. $1.5,3$ and 10 times critical power $P_{c r}$ which is $9 \mathrm{kw}$ for the wavelength $\lambda=$ $1500 \mathrm{~nm}$.Using the boundary condition $\alpha=\alpha_{0}$ and $\beta=$ $\beta_{0}$ at $\mathrm{z}=0$,for values of $\alpha_{0}=.02 \mathrm{~cm}, \beta_{0}=.015 \mathrm{~cm}$ predict an exponential decrease in $\alpha$ and $\beta$ which approaches zero over a finite propagation distance. i.e. when $P_{i n}>P_{c r}$ the width of an elliptical beam collapses over a finite propagation distance which for $1.5,3$ and 10 times $P_{c r}$ is $8.3 \mathrm{~cm}, 6.3 \mathrm{~cm}$ and $4 \mathrm{~cm}$, respectively. However, from Eqns. (12) and (13) a necessary condition for self-trapping can be written as

$$
\frac{d^{2} \alpha}{d z^{2}}=0=\frac{d^{2} \beta}{d z^{2}} \text { at } z=z_{s f}
$$

Where $z_{s f}$ is self-focusing length. It implies that at $z_{s f}, \alpha$ $=\beta$ which is a condition at self-focusing. The values of $z_{s f}$ thus determined from the derived condition are $7.1 \mathrm{~cm}, 5.6$ $\mathrm{cm}$ and $3.0 \mathrm{~cm}$ for $1.5,3$ and 10 times $P_{c r}$, respectively. In these solutions value of $\mathrm{k}$ was taken as

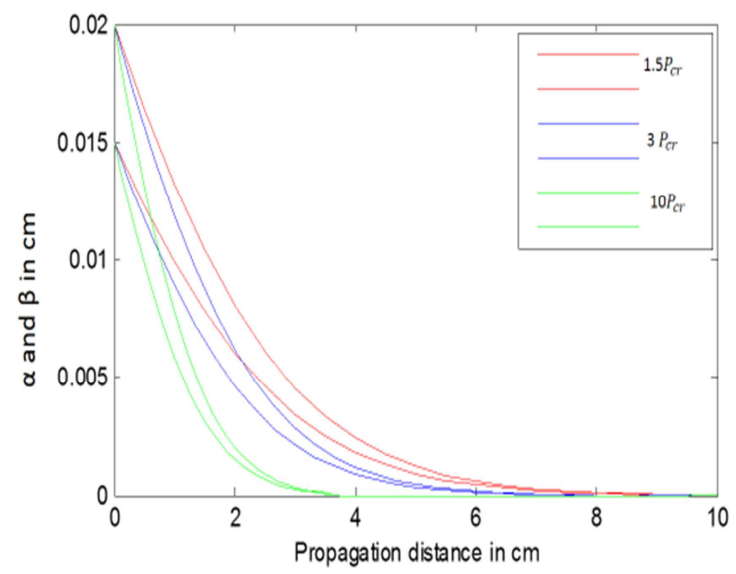

Figure 1. Variation of beam widths $\alpha$ and $\beta$ with propagation distance for input power 1.5, 3, 10 times the critical power for $\alpha_{0}=.02, \beta_{0}=.015$.

$1.4 \times 10^{3} \mathrm{~cm}^{-1}[28,29]$ and the wavelength of propagation as $1500 \mathrm{~nm}$ - the 'eye-safe region' wavelenth in optical communication.It is observed from Fig. 1 that the beam width collapses more rapidly with input power and the collapse distance decreases with increase in input power of the Gaussian beam. The collapse distance scales as $\frac{1}{\sqrt{P}}$ for $\mathrm{P} \gg P_{c r}$ [30] but at higher powers collapse scales as $\frac{1}{P}[31,33]$.Collapse occurs when the whole beam shrinks to a point. Further, a collapsing beam exhibits sharp rise in intensity [32]. The intensity of the propagating beam at three input powers in the present case increases on reaching $z_{\mathrm{sf}}$. But, the rise in intensity of the beam with input power $10 P_{c r}$ only bears signature of a collapsing beam during selffocusing. The variation of intensity with propagation distance is presented in Figure 2 and is plotted using Eqn.(11). The values of $H_{0}$ for the three input powers are $0.1974 \times 10^{-10}$, $0.3948 \times 10^{-10}$ and $0.1316 \times 10^{-9}$ (in c.g.s).

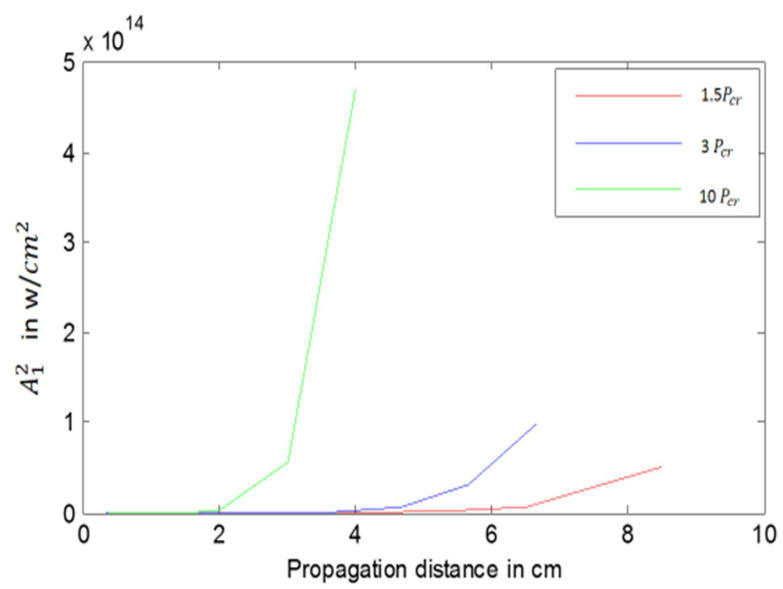

Figure 2. The variation of $A_{1}^{2}(z)$ with propagation distance at input power 1.5P $P_{c r}, 3 P_{c r}$ and $10 P_{c r}$.

The threshold power for collapse of a beam for an elliptical beam is approximated as $P_{t h}(e) \approx$ $\left[0.4 \frac{e+1 / e}{2}+0.6\right]$ times the threshold power for circular beam, $P_{t h}(e=1)$.Here e $=\beta / \alpha$ [34].The threshold power for collapse increases with input beam ellipticity and the threshold power for collapse is above the critical power (35).

Using the boundary condition at $\mathrm{z}=0, \alpha=\alpha_{0}$ and $\beta=\beta_{0}$, the solutions for Equations (6) and (7) are given by,

$$
\begin{aligned}
& c_{1}(\mathrm{z})=-\frac{\mathrm{k}}{\sqrt{2 \alpha(Z)}} \sqrt{\left\{H_{0} \ln \left(\frac{\alpha(z)}{\alpha_{0}}\right)\right\}}, \\
& c_{2}(\mathrm{z})=-\frac{\mathrm{k}}{\sqrt{2 \beta(Z)}} \sqrt{\left\{H_{0} \ln \left(\frac{\beta(Z)}{\beta_{0}}\right)\right\}}
\end{aligned}
$$

The variation of $\mathrm{x}$ and $\mathrm{y}$ direction curvature $c_{1}$ and $c_{2}$ with propagation distance at input power $1.5 P_{c r}, 3 P_{c r}$ and $10 P_{c r}$ is shown in figre(3) and (4).

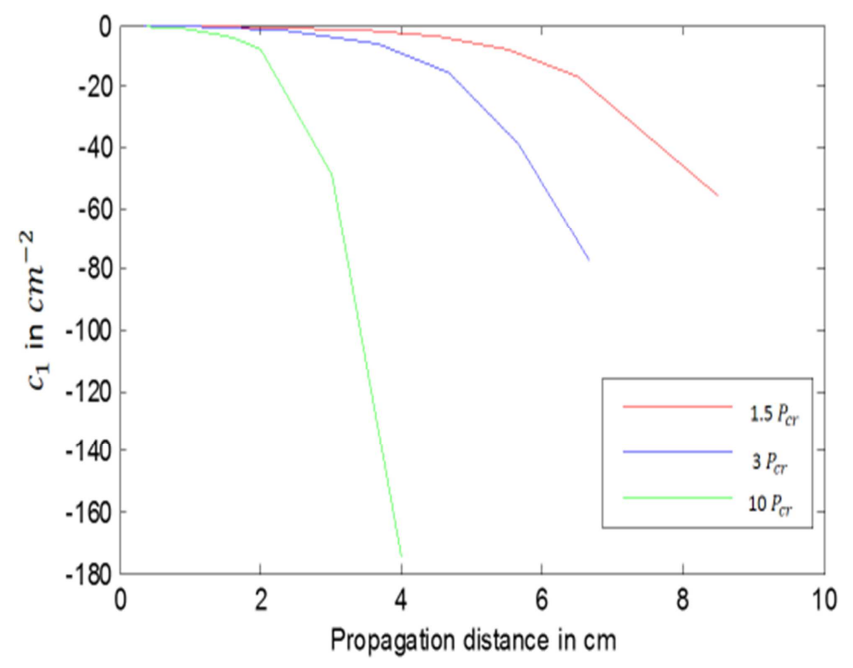

Figure 3. Variation of curvature $c_{1}$ with distance of propagation for input power 1.5, 3, 10 times $P_{c r}$. 


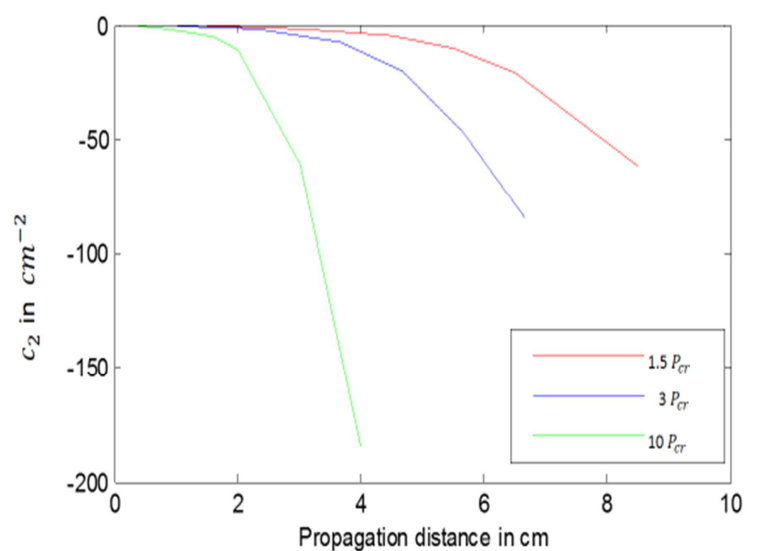

Figure 4. Variation of curvature $c_{2}$ with distance of propagation for input power 1.5, 3, 10 times the critical power.

Evolution of both the curvatures $c_{1}$ and $c_{2}$ shows a decreasing trend with propagation distance and falls of rapidly around half the collapse distance. The variations are accentuated as input power increases. The curvatures diminish three folds more for 8.5 times increase in input power on propagating $4.3 \mathrm{~cm}$ less. The negative sign in the magnitude of $c_{1}$ and $c_{2}$ is indicative of the decrease in curvatures with propagation distance i.e convergent point, the radius of the wave front of the transmitted beam is negative[36].

Again, using the boundary condition , at $\mathrm{z}=0, \alpha=\alpha_{0}$ and $\beta=\beta_{0}$, the solution of equation ( 8 ) is given by,

$$
\phi(\mathrm{z})=\frac{1}{4 k}\left[\int_{\alpha_{0}}^{\alpha} \frac{d z}{\alpha^{2}}+\int_{\beta_{0}}^{\beta} \frac{d z}{\beta^{2}}\right]
$$

Figure 5 displays the variation of longitudinal phase $\phi$ as a function of propagation distance for input power 1.5, 3, 10 times $\mathrm{P}_{\mathrm{cr}}$. It is observed that phase $\phi(\mathrm{z})$ is negative and for the beam with $P_{i n}=10 P_{c r}$ phase changes abruptly at $\mathrm{z}_{\mathrm{sf}}$. It should be noted that the longitudinal phase may be positive or negative depending on the value of intensity parameters[37].

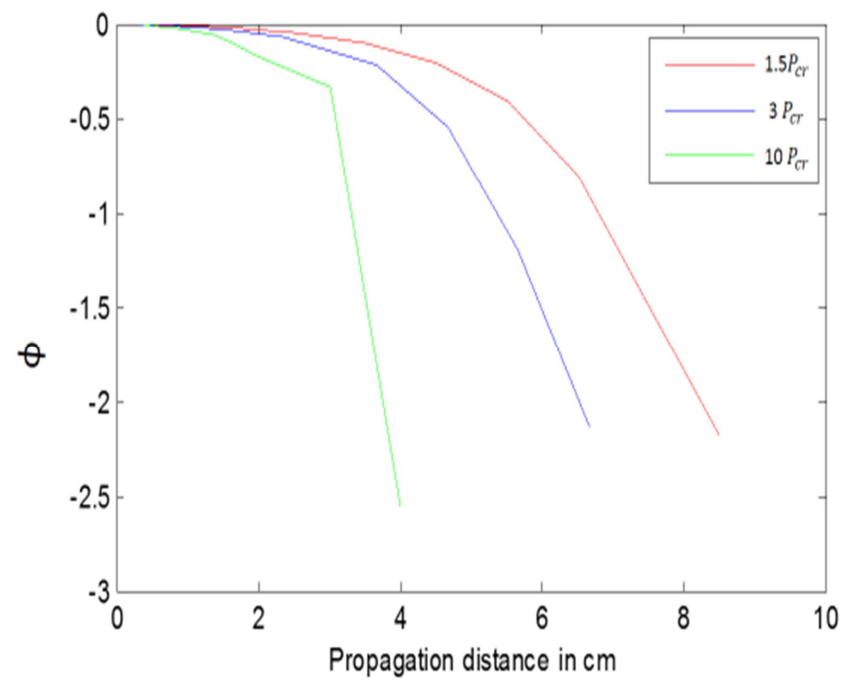

Figure 5. Variation of longitudinal phase $\phi$ with distance of propagation for input power 1.5,3 and 10 times the critical power.

\section{Conclusion}

The evolution of the beam width,curvature and phase was analysed by variational approach. The solutions obtained using finite difference scheme based on fixed point iteration predict stationary self-focussing for elliptical Gaussian beam propagating in chalcogenide glass with distinct beam collapse evident at $10 \mathrm{P}_{\mathrm{cr}}$ input power. The variations in curvature, phase and intensity were more accentuated for beam with input power of $10 \mathrm{P}_{\mathrm{cr}}$ compared to those in input power of $1.5 \mathrm{P}_{\mathrm{cr}}$ and $3 \mathrm{P}_{\mathrm{cr}}$ and occurs as the beam approches self-focusing length or at self-focus length.It is indicative that self-action effects are more effective at input power of $10 \mathrm{P}_{\mathrm{cr}}$. The change of phase is negative with distance of propagation. The present study is significant for optical communication leading to transfer of energy in narrow beams without losses at low optical power(for silica fibre, $P_{c r}=2.1 \mathrm{MW}$ )[38]. Further, the study of beam parameters at self focusing distances also helps in material selection and material modification in the fibre optic communications, because properly selecting beam parameters, like power,wavelength and Kerr nonlinear material can prevent the damage of fibre and waveguide etc.

\section{References}

[1] R.W. Boyd (eds), Self-focusing : Past and Present, Topics in Applied Physics, 2009, Ch.4 Springer Science.

[2] Report to A.P.S. of the Study Group on Science and Technology of Directed Energy Weapons, Rev. Mod. Phys,(1987), 59, 589.

[3] S. E. Bodner, D. G. Colombant, J H. Gardner, R. H Lehmberg, S. P. Obenschain, L. Phillips, A .J Schmitt, J. D. Sethian, R. L. McCrory, W. Seka, C. P. Verdon, J. P. Knauer, B. B. Afeyan and H. T .Powell, Phys. Plasmas, 1998, 5, 1901.

[4] P. M. Lushnikov and N. Vladimirova, Nonlinear combining of laser beams, arXiv: 1404. 1050v1 [nlin.PS], 2014.

[5] P. M. Lushnikov, S. A. Dyachenko, and N. Vladimirova, Beyond leading-order logarithmic scaling in the catastrophic self focusing of a laser beam in kerr media, Phys. Rev. A,2013, 88,013845 .

[6] N. Berti, W. Ettoumi,J. Kasparian, and J.-P.Wolf, Reversibility of laser filamentation, Optics express,2014, 21062.

[7] I. Kubat, C. R. Petersen, U. V. Møller, A. Seddon, T. Benson, L. Brilland, D. M'echin,P. M. Moselund, and O.Bang, Thulium pumped mid-infrared 0.9-9 $\mu \mathrm{m}$ supercontinuum generation inconcatenated fluoride and chalcogenide glass fibers,Optics express, 2014, 3959.

[8] R.Vyas and V. F. Gupta, Appl. Opt.1988, 27, 4701.

[9] P. A. Konyaev and V. P. Lukin, Sov. J. Quantum Elect, 1988, 18, 217.

[10] V. V. Semak and M. N. Shneider, Splendeurs et misères de theory of laser beam propagation in nonlinear media,2014, arXiv:1404.3744, Cornell University Library.

[11] R Y Chio, E Garmire and C H Townes, Phys. Rev. Lett,1964, 13,479 . 
[12] M Hercher, Beam propagation in transparent media, J. Opt. Soc. Am, 1964, 54, 563.

[13] G.A. Askaryan, Effects of the gradient of a strong electromagnetic beam on electrons and atoms, Sov. Phys. JETP 1962,15, 1088-1090 (transl. Zh. Eksp. i Teor. Fiz. 42, 1672.)

[14] J. A. Fleck, Jr., and P. L. Kelley, Temporal aspect of the selffocusing of optical beams, Appl. Phys. Lett,1969,15, 313.

[15] M.D. Feit, and J. A. Fleck, Jr., Beam nonparaxiality, filament formation, and beam breakup in the self-focusing of optical beams, J. Opt. Soc. Am, 1988,B 5, 633.

[16] K. D. Moll, A. L. Gaeta, and G. Fibich, Self-similar optical wave collapse: observation of the townes profile, Phys. Rev. Lett, 2003, 90, 203902-1.

[17] G. Fibich, W. Ren, and X.P. Wang, Numerical simulations of self-focusing of ultrafast laser pulses, Phys. Rev. E, 2003, 67, 056603 .

[18] R. M. Joseph, A. Taflove, Spatial soliton deflection mechanism indicated by FDTD Maxwell's equations modeling, IEEE Photon. Tech. Lett,1994, 6, 1251.

[19] R. W. Ziolkowski, and J. B. Judkins, Full-wave vector Maxwell equation modeling of the self-focusing of ultra-short optical pulses in a nonlinear Kerr medium exhibiting a finite response time, J. Opt. Soc. Am. B, 1993,10, 186.

[20] A.Couairon, A.Mysyrowicz, Femtosecond Filamentation in Transparent Media, Physics Reports, 2007,441, 47.

[21] Y. Sivan, G. Fibich, B. Ilan, and M. I. Weinstein, Qualitative and quantitative analysis of stability and instability dynamics of positive lattice solitons, Physical Review,2008, doi:10.1103/E.78.046602

[22] V.V. Semak and M.N. Shneider, Effect of power losses on self-focusing of high intensity laser beam in gases and role of plasma in generation of multiple filaments, J. Phys. D: Appl. Phys,2013, 46 185502 doi:10.1088/0022-3727/46/18/185502

[23] J. T. Gopinath, M. Soljacic, E.P. Ippen, V.N. Fuflyigin, W.A. King and M. Shurgalin, Third order nonlinearities in Ge-AsSe-based glasses for telecommunications applications, J. Appl. Phys,2004, 96, 6931-6933.

[24] S. Sagadevan, E.Chandraseelan, Applications of chalcogenide glasses : An overview,IJCTR,2014,6,11.
[25] D Anderson, M Bonnedal and M Lisak, Phys. Fluids, 1979, 22, 1838.

[26] Anderson, D. and M. Bonnedal, Variational approach to nonlinear self-focusing of Gaussian laser beams, Phys. Fluids, 1979, Vol. 22, 105-109.

[27] J. Requejo-Isidro , A.K. Mairaj, V. Pruneri , D.W. Hewak, M.C. Netti ,J.J.Baumberg, Self refractive non-linearities in chalcogenide based glasses, Journal of Non crystalline solids, 2003,317,241-246.

[28] R A Meyers, Encyclopedia of physical science and technology1987, 7, 189.

[29] P Das, Lasers and Optical Engg, Narosa Publ. House, New Delhi,1991.

[30] A L Schawlow, Lasers and light, W H Freeman \& Co,1969.

[31] P.L. Kelley, Self-focusing of optical beams. Phys. Rev. Lett, $1965,15,1005-1008$.

[32] R.Y.Chiao, T. K. Gustafson, and P. L. Kelley, Self-Focusing of Optical Beams; Springer series: Topics in appl.Physics,vol114,Springer,NY,Editors:R.W.Boyd,

S.G.Lukishova,Y.R.Shen,2009;605 p.illus,ISBN:978-0-38732147-9.

[33] G. Fibich, S. Eisenmann, B. Ilan, Y. Erlich, M. Fraenkel, Z. Henis, A.L. Gaeta, and A. Zigler, Self-focusing distance of very high power laser pulses, Opt. Express, 2005; 13, 58975903.

[34] G. Fibich and B. Ilan, Self-focusing of elliptic beams: An example of the failure of the aberration less approximation, JOSA B, 2000; 17, 1749-1758.

[35] G. Fibich, Some modern aspects of self-focusing theory, Selffocusing : Past and Present, Topics in Applied Physics, Ch.17, Springer Science,2009;114, 413-438.

[36] B.E.A. Saleh and M.C. Carl Teich, Fundamental of Photonics, beam optics,chapter3,JohnWiley\&Sons, Inc,1991.

[37] R. Kaur, T. Gill and R. Mahajan, Self-focusing, selfmodulation and stability properties of laser beam propagating in plasma: A variational approach, Journal of Physics: Conference Series, 2010; 208.

[38] R. K. Khanna and P. K. Nagar, self-focusing of Gaussian laser beam in parabolic profile optical fiber having Kerr nonlinearity, IJPAP, 2001, 39. 